\title{
Avaliação do incremento em volume de madeira de Quassia amara L.- Simaroubaceae, em cultivo agroecológico no trópico úmido da Costa Rica
}

\author{
PRÓSPERO, R. ${ }^{1}$; MING, L.C. ${ }^{1 *}$; ROJAS, R.D. ${ }^{2}$; OCAMPO, R.A. ${ }^{2}$ \\ 'Departamento de Produção Vegetal - Horticultura. Faculdade de Ciências Agronômicas - UNESP, Caixa Postal \\ 237, Rua José Barbosa de Barros, no 1780, CEP: 18610-307, Botucatu-Brasil. rodrigo_prospero@ig.com.br; * \\ linming@fca.unesp.br ${ }^{2}$ Empresa Bougainvillea S.A. Santo Domingo, Apartado Postal 764-3100, Heredia-Costa \\ Ricaquassia@racsa.co.crbougainvillea@ice.co.cr
}

\begin{abstract}
RESUMO: Quassia amara é arbusto de 3 a 6 metros de altura, tendo sido retirado indiscriminadamente das florestas para extrair do caule as quassinas usadas na indústria farmacêutica e como inseticida em agricultura orgânica. Não se tem muita informação técnica acerca do crescimento desta espécie para subsidiar estratégias de manejo sustentado. Este trabalho tem como objetivo avaliar o crescimento de $Q$. amara L. em cultivo agroecológico na Costa Rica. O trabalho consistiu em realizar avaliações do desenvolvimento de indivíduos de $Q$. amara em parcelas permanentes de medições, instaladas em meio às plantações desta espécie em consórcio com essências arbóreas. Foram efetuadas medições de diâmetro do caule a 10 $\mathrm{cm}$ do solo e altura total. Foi observado que em função das taxas de crescimento vegetal e incrementos médio e corrente anuais (IMA e ICA), mesmo após cinco anos de plantio, a madeira de Quassia amara para extração de quassinas não está pronta para colheita.
\end{abstract}

Palavras-chave: domesticação, Quassia amara, Simaroubaceae, Costa Rica, plantas medicinais

\begin{abstract}
Evaluation of wood volume increment in Quassia amara L. - Simaroubaceae, under agroecological cultivation in the humid tropic of Costa Rica. Quassia amara is a 3$6 \mathrm{~m}$ tall bush which has been indiscriminately removed from forests for extraction of quassins from its stem. These substances have been used in pharmaceutical industry and as insecticide in organic agriculture. There is not much technical information about the growth of this species to subsidize sustainable management strategies. The aim of this study was to evaluate the growth of Quassia amara under agroecological cultivation in Costa Rica. The study consisted in evaluating the development of $Q$. amara individuals in permanent plots intercropped with other tree species. Stem diameter at $10 \mathrm{~cm}$ from the ground and total height were measured. According to the results concerning growth rates, Mean Annual Increment (MAI) and Current Annual Increment (CAl), even at five years after planting, $Q$. amara wood is not ready to be harvested.
\end{abstract}

Key words: domestication, Quassia amara, Simaroubaceae, Costa Rica, medicinal plants

\section{INTRODUÇÃO}

A região da América Central conta com grande reserva de cobertura florestal primária de aproximadamente 17,4 milhões de hectares (36\% da área total). Destes, cerca de 11 milhões de hectares têm potencial para aproveitamento industrial sustentável, enquanto que o restante, ao redor de 6.5 milhões de hectares, poderia ser dedicado à conservação da biodiversidade e desenvolvimento do turismo (Finegan et al., 1993).

A perda de recursos naturais tem gerado preocupação tanto do lado governamental, quanto do lado não governamental acerca da importância de se manejar de forma consciente estes recursos para garantir a perpetuidade. As florestas tropicais são as que mais sofrem com essas perdas. Segundo Alfaro (1996), na década entre 1980-1990, os bosques

Recebido para publicação em 28/04/2008

Aceito para publicação em 24/07/2009 
tropicais foram desmatados a uma taxa de $0.9 \%$ ao ano. Budowski (1995) diz que a perda de diversidade está diretamente relacionada com o desmatamento e nas florestas tropicais úmidas se encontram mais ou menos $50 \%$ de toda a diversidade do mundo. No entanto, segundo o mesmo autor, valorizar a biodiversidade implica em conhecêla primeiro.

Foi determinado em um diagnóstico de Costa Rica, feito por Ocampo (1994), que se aproveitam um total de 406 espécies medicinais e que se comercializam 133 espécies, das quais 37\% são plantas silvestres. Atualmente a pressão pelo uso destas espécies vem aumentando devido à crescente procura e resultante aumento na fabricação de produtos fitoterápicos ou naturais.

O manejo sustentável busca satisfazer as necessidades do presente sem comprometer a capacidade das gerações futuras de atenderem as próprias necessidades. Para se ter o manejo sustentável dos recursos naturais é necessário reunir suficientes conhecimentos sobre ecologia e silvicultura das florestas tropicais, que permitam proteger as funções, ecossistemas e biodiversidade, e que além da exploração da madeira, se busquem outros benefícios que não madeireiros das plantas. É necessário assumir responsabilidades de ordem teórica e social para garantir a sustentabilidade do uso dos recursos naturais (Putz, 1994).

A taxa de desmatamento na Costa Rica é de $600 \mathrm{Km}^{2} \mathrm{ano}^{-1}$, dentre os quais se encontram materiais para artesanato, plantas com potencial farmacêutico, biocidas, curativos e outros. Devido ao desaparecimento ou escassez destes recursos genéticos, instituições nacionais e internacionais têm se dedicado à tarefa de promover o aproveitamento integral dos recursos florestais, tendo como uma destas medidas o uso dos chamados produtos não madeireiros das florestas (Gonzáles, 1997).

Os produtos não madeireiros podem ser manejados juntamente com os produtos madeireiros, incrementando a produtividade e rentabilidade das florestas. Alguns destes produtos podem ser cultivados em sistemas agroflorestais ou até em monocultivo. Podem ser extraídos de maneira sustentável, sem causar desmatamento ou dano irreversível ao meio ambiente e a biodiversidade. Em geral, têm fortes laços e se complementam com os componentes de desenvolvimento sustentável e com as atividades ambientalmente adequadas, especialmente nas áreas rurais (FAO, 1995).

Quassia amara é considerado produto não madeireiro com grande potencial de manejo por possuir algumas características interessantes, como a capacidade de rebrota após a colheita e adaptabilidade a solos com variado $\mathrm{pH}$ (Guzmán, 2000).

Quassia amara é arbusto de 3 a 6 metros, que pertence à família Simaroubaceae, com folhas de pecíolo alado. Na floresta mostra uma dominância apical expressada pela formação de um eixo principal, que está freqüentemente danificado no ápice por ataque de insetos (Brown, 1995). Esse dano no ápice faz com que a planta produza indivíduos cujo eixo principal se divide em dois ou três a uma altura variável. Cada um desses eixos possui um crescimento predominantemente ortotrópico (Villalobos, 1995).

Os princípios ativos desta espécie são basicamente as quassinas, que são utilizadas em inseticidas naturais na agricultura orgânica, além de serem empregadas na indústria farmacêutica na produção de medicamentos relacionados a dores de estômago (Ocampo, 1994).

Não existem ainda estudos visando verificar os índices de crescimento desta espécie, para que uma proposta de manejo sustentável seja desenvolvida por agricultores, visando obtenção desse material vegetal em sistema agroecológico de cultivo.

Este trabalho teve como objetivo avaliar o crescimento em diâmetro e altura de Quassia amara em cultivo agroecológico na Costa Rica, avaliandose a melhor época de corte para aproveitamento comercial da madeira para fins industriais/medicinais.

\section{MATERIAL E MÉTODO}

O trabalho de campo consistiu em realizar avaliações em parcelas permanentes de medições instaladas em meio às plantações de $Q$. amara, localizadas em Baltimore, Matina, província de Limón, no lado caribenho de Costa Rica, com coordenadas geográficas N100 01' 03.1" e W83ํ1' 41.6”. Essas parcelas possuem cinco anos de histórico de medições dos diâmetros a $10 \mathrm{~cm}$ do solo e alturas totais.

Iniciou-se a plantação de sementes das parcelas permanentes de medições em fevereiro/ março de 2001. Nesta etapa foram utilizadas caixas de areia como germinadores. As sementes foram provenientes da Zona de Baja Talamanca, mais precisamente na Reserva Indígena Kekoldi, província de Limón, Costa Rica.

Após o enraizamento completo e prédesenvolvimento em canteiros de terra adubada (outubro do mesmo ano), as plântulas foram levadas para o plantio em campo. Para este transplante, as plantas tiveram toda parte aérea cortada a cerca de $30 \mathrm{~cm}$ do solo e a raízes expostas quando retiradas do solo. A plantação foi conduzida em área de aproximadamente 4 ha em sistema arborizado, composto principalmente por Cordia alliodora (Ruiz \& Pav.) Oken (Boraginaceae), Theobroma cacao L. (Sterculiaceae) e Bactris gasipaes Kunth. (Arecaceae). Estas plantações foram efetuadas em espaçamentos que variam de $2 \times 2$ a 1,5 x 1,5 metros.

Foram demarcadas seis parcelas para medições permanentes de crescimento no mesmo 
ano de plantação das mudas de Quassia amara em campo (2002). Estas parcelas possuíam $10 \times 10$ metros com 25 a 44 plantas de $Q$. amara e foram dispostas aleatoriamente no campo experimental.

Todas as medições foram efetuadas entre os meses de março e abril de 2002 a 2006. Em cada individuo de Quassia amara foram medidos os diâmetros a $10 \mathrm{~cm}$ da superfície do solo e as alturas totais. As alturas totais foram medidas com fita métrica da base da planta em contato com o solo até a ponta do eixo de maior tamanho e os diâmetros com paquímetro comum.

Foram calculados o Incremento Corrente Anual (ICA) e Incremento Médio Anual (IMA) em volume $\left(\mathrm{m}^{3}\right)$. Denomina-se ICA o crescimento em volume ocorrido no período de um ano, e IMA ao resultado da divisão do volume pela idade da floresta. Deve ser notado que a curva de ICA atinge o máximo antes da curva de IMA, e que as duas curvas se cruzam no ponto de máximo IMA. Determinar a idade ótima de corte através deste critério implica, portanto, no corte da floresta quando esta atingir a idade de máximo IMA (Rodrigues, 1991).

Para cálculo de ICA e IMA foram usadas as seguintes fórmulas:

\section{Sendo:}

- IMA = V / t

IMA = Incremento Médio Anual $\left(\mathrm{cm}^{3}\right)$;

$\mathrm{V}=$ Volume $\left(\mathrm{cm}^{3}\right)$ produzido em função da idade t (anos);

$\mathrm{t}=$ Idade da plantação (anos).

$$
\begin{aligned}
& \text { - ICA = V - V' } \\
& \text { Sendo: } \\
& \text { ICA = Incremento Corrente Anual }\left(\mathrm{cm}^{3}\right) ; \\
& \text { V = Volume }\left(\mathrm{cm}^{3}\right) \text { medido no ano atual; } \\
& \text { V'= Volume }\left(\mathrm{cm}^{3}\right) \text { medido no ano anterior. }
\end{aligned}
$$

Obs: todos os volumes foram calculados com base na seguinte fórmula, com fator de correção ( $f$ ) de 0,5:

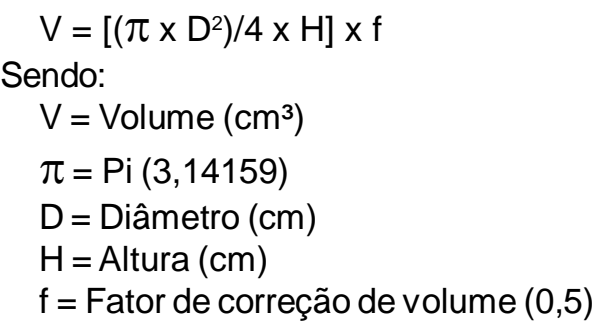

\section{RESULTADO E DISCUSSÃO}

As parcelas possuem diferentes médias de incrementos em volume de madeira, como pode ser observado na Tabela 1. A parcela 1 apresentou a maior média de volume, com estimativa de $1545 \mathrm{~cm}^{3}$.

$\mathrm{Na}$ Tabela 2 são apresentados dados de Incrementos Médios Anuais (IMA) e Incrementos Correntes Anuais (ICA) em volume $\left(\mathrm{cm}^{3}\right)$ das seis parcelas. O primeiro ano do plantio (2002 a 2003) foi o que demonstrou menores taxas de Incrementos Médios e Correntes Anuais. A partir do 2a ano (2003) os dados de ICA começam a ter maiores taxas de incremento. Já no ano de 2006 observa-se o início de queda no ICA em volume $\left(\mathrm{cm}^{3}\right)$.

O ICA mostra o incremento em dado período de tempo na(s) variável(eis) estipulada(s) e pode mostrar quando o desenvolvimento da planta se estagna ou começa a entrar em declínio. Quando este dado de Incremento Corrente Anual decresce significa, neste caso estudado, que a planta possivelmente já está começando a se tornar "madura", e que deste ponto em diante pode continuar a apresentar quedas na taxa de crescimento. Essas menores taxas de Incrementos não são economicamente interessantes, já que significa menores incrementos em volume $\left(\mathrm{cm}^{3}\right)$ de madeira aproveitável por ano.

Na Figura 1 é apresentada relação entre 0 Incremento Médio e Corrente Anual (IMAe ICA) para volume $\left(\mathrm{cm}^{3}\right)$. O ponto ótimo comercial de colheita se dá quando as linhas de incremento médio e corrente anual se cruzam, o que até o momento não

TABELA 1. Evolução de incremento de indivíduos de Quassia amara (em volume - $\mathrm{cm}^{3}$ ), durante 5 anos de medição em Baltimore, Matina, província de Limón, Costa Rica.

\begin{tabular}{cccccc}
\hline & \multicolumn{5}{c}{ Ano } \\
\cline { 2 - 6 } Parcela & 2002 & 2003 & 2004 & 2005 & 2006 \\
\hline 1 & 5,66 & 12,55 & 194,08 & 764,39 & 1545,01 \\
2 & 4,84 & 44,22 & 268,83 & 651,56 & 1243,01 \\
4 & 1,78 & 9,50 & 91,63 & 426,37 & 644,81 \\
5 & 6,17 & 17,44 & 131,52 & 492,13 & 1046,03 \\
6 & 1,82 & 8,22 & 55,17 & 551,13 & 565,26 \\
7 & 3,18 & 35,45 & 282,49 & 811,95 & 1196,33 \\
\hline Média & 3,91 & 21,23 & 170,62 & 616,25 & 1040,07 \\
\hline
\end{tabular}

Rev. Bras. PI. Med., Botucatu, v.11, n.4, p.450-454, 2009. 
TABELA2. Incremento Corrente Anual (ICA) e Incremento Médio Anual (IMA) dos volumes ( $\left.\mathrm{cm}^{3}\right)$ das seis parcelas de medições localizadas em Baltimore, Matina, província de Limón, Costa Rica.

\begin{tabular}{rrrrrr}
\hline & \multicolumn{5}{c}{ Ano } \\
\cline { 2 - 6 } & $\mathbf{2 0 0 2}$ & $\mathbf{2 0 0 3}$ & $\mathbf{2 0 0 4}$ & $\mathbf{2 0 0 5}$ & $\mathbf{2 0 0 6}$ \\
\hline IMA & 3,9 & 10,6 & 56,9 & 154,1 & 208,0 \\
ICA & 7,8 & 17,3 & 149,4 & 445,6 & 423,8 \\
\hline
\end{tabular}

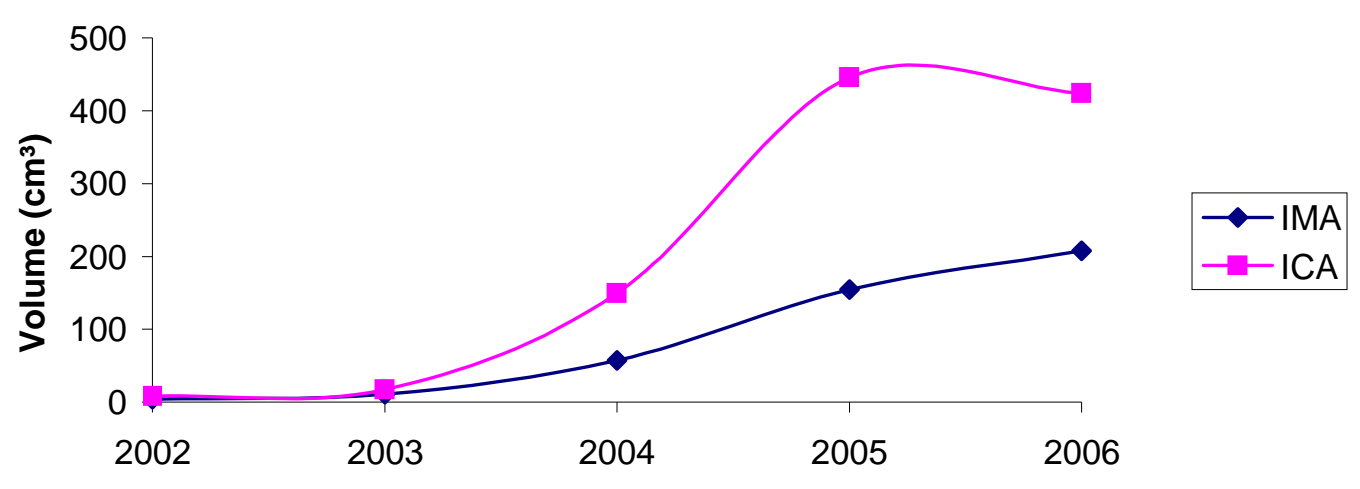

Anos

FIGURA 1. Incremento Médio Anual (IMA) e Incremento Corrente Anual (ICA) de volume ( $\left.\mathrm{cm}^{3}\right)$ estimado com base em diâmetro (a $10 \mathrm{~cm}$ do solo) em mm, mostrando evolução destes índices durante os 5 anos de medições.

aconteceu nas parcelas estudadas.

Pode-se verificar que após o primeiro ano de plantio em campo (2002) e até o segundo ano (2003), não houve aumento nos incrementos, como pode ser observado na Figura 1. Este fato ocorre seguramente em função do stress que a planta sofreu ao ser transplantada do viveiro para o campo e posterior adaptação ao novo ambiente. Este processo de transplante da planta do viveiro ao campo se deu eliminando todas as folhas da planta com corte a mais ou menos $30 \mathrm{~cm}$ do solo e tirando-as do solo dos canteiros do viveiro e passando diretamente para campo.

Com os dados dendrométricos obtidos ao longo dos 5 anos de medições nas Parcelas Permanentes de Medições, pode-se concluir que a colheita da planta Quassia amara ainda não pode ser realizada considerando-se incrementos em volume para ter maior aproveitamento econômico da cultura, já que as taxas de ICA ainda estão superiores as taxas de IMA. Além disso, é preciso aprofundar o estudo em concentrações de quassinas durante 0 crescimento desta planta para estabelecer o ponto ótimo de colheita levando em consideração o ativo a ser utilizado da planta.

\section{REFERÊNCIA}

ALFARO, M. Aspectos relevantes del manejo y conservación de los recursos naturales: Presente y futuro. In: CURSO DE ORDENACIÓN FORESTAL, Heredia, Costa Rica. Resumen ... Heredia: Escuela de Ciencias Ambientales, Universidad Nacional, 1996. p.13.

BROWN, N.R. The autoecology and agroforestry potential of the biterwood tree Quassia amara L. Ex Blom (Simaroubaceae). 1995. 250p. Dissertação (Ph.DÁrea de Concentração de Ciências das Plantas)Colégio de Agricultura e Ciências da Vida, Cornell University, New York.

BUDOWISKI, G. La biodiversidad y el manejo de los recursos naturales. In: OCAMPO, $R$. (Ed.). Potencial de Quassia amara como insecticida natural. Turrialba: CATIE, 1995. p.3-8. Informe técnico n.267.

FAO. State of the World's Forest. Electronic publishing policy and support branch of food and agriculture organization of the United Nations. 1995. Disponível em: <http://www.fao.org>. Acesso em: 15 mai. 2008.

FINEGAN, B.; REICHE, C.; HUTCHINSON, I. Los bosques húmedos de América Central: Su manejo sostenible es posible y rentable. Revista Forestal Centroamericana, v.2, n.6, p.17-27, 1993.

GUZMÁN, V.M. Evaluación del crecimiento de Hombre Grande (Quassia amara) como respuesta a la poda en “Baja Talamanca”, Limón, Costa Rica. 2000. 79p. 
Trabalho de Conclusão de Curso (Graduação em Engenheira Florestal) - Facultad de la Tierra y Mar, Universidad Nacional, Heredia, Costa Rica.

OCAMPO, R. Estado de la conservación de hombre grande (Quassia amara) en Centroamérica: un insecticida potencial en el Neotropico. In: ENCUENTRO LATINOAMERICANO Y DEL CARIBE SOBRE NIM (Azadirachta indica) Y OTROS INSECTICIDAS VEGETALES, 1994, República Dominicana. Anales... República Dominicana, 1994. p.10-20.

GONZALES, R.M. Estudio de sistemas de producción de Hombre Grande (Quassia amara) en la región huetar norte. 1997. 86p. Trabalho de Conclusão de Curso (Graduação em Engenheira Agronômica) - Instituto Tecnológico de Costa Rica, San Carlos.
PUTZ, F.E. Aproaches to sustainable forest Management. Working paper CIFOR, n.267, p.11-13, 1994. Disponível em: <http://www.cifor.cgiar.org>. Acesso em: 15 mai. 2008. RODRIGUES, L.C.E. Gerenciamento da produção florestal. Documentos Florestais. Piracicaba: Universidade de Sao Paulo, Escola Superior de Agricultura Luiz de Queiroz, Departamento de Ciências Florestais, 1991, v.13, p.1-41.

VILLALOBOS, R.S. Distribución de Quassia amara L. ex Blom en Costa Rica, Y su relación con los contenidos de cuasina y neocuasina (insecticidas naturales) en su tejidos. 1995. 92p. Dissertação (Mestrado - Área de concentração: Manejo y Conservación de Bosques Tropicales y Biodiversidad) - Centro Agronómico Tropical de Investigación y Enseñanza, Turrialba. 